\title{
Estudo epidemiológico da febre purpúrica brasileira - Epidemia em localidade do Estado de São Paulo (Brasil), 1986*.
}

\section{Brazilian purpuric fever - An epidemiological study of an outbreak in the locality of the S. Paulo State, Brazil, in 1986.}

\author{
Ligia R.S. Kerr-Pontes**, Antonio Ruffino-Netto**
}

\begin{abstract}
KERR-PONTES, L.R.S. \& RUFFINO-NETTO, A. Estudo epidemiológico da febre purpúrica brasileira - Epidemia em localidade do Estado de São Paulo (Brasil), 1986. Rev. Saúde públ., S. Paulo, 25: 375-80, 1991. Estudou-se um surto de febre purpúrica brasileira ocorrido em Serrana, SP (Brasil) em 1986 e sua associação com conjuntivite purulenta, aglomeração e sintomas respiratórios. Foi adotado o modelo de estudo, caso-controle. Chamou-se caso confirmado o paciente que satisfizesse um conjunto de critérios tendo "score" maior ou igual a 12 pontos, e caso suspeito "score" entre 8 e 12 (o "score" foi efetuado usando-se o seguinte critério: ocorrência de febre, igual a 5,pontos; diarréia e/ou vômitos igual a 1; fenômenos hemorrágicos igual a 3; plaquetopenia e/ou leucopenia igual a 3; hemocultura e/ou líquor e/ ou cultura de orofaringe positiva para Haemophylus aegyptius igual a 7; síndrome de Waterhouse Friedrichsen igual a 7). Tomou-se como controle crianças com "score" menor do que 5 . O controle foi pareado com o caso segundo as variáveis idade, sexo e condição sócio-econômica. Levantaram-se informações sobre 14 casos confirmados, 38 suspeitos e 78 controles. Concluiu-se que a febre purpúrica brasileira apresentou forte associação com conjuntivite purulenta pregressa e/ou atual; parece haver associação entre aglomeração e febre purpúrica e que os sintomas respiratórios como tosse e/ou coriza não estão a ela associados, pelo menos na população estudada.
\end{abstract}

Descritores: Síndrome de Waterhouse-Friedrichsen, epidemiologia. Surtos de doenças.

\section{Introdução}

A Febre Purpúrica Brasileira (FPB) foi descrita pela primeira vez na localidade de Promissão-SP, por ocasião de um surto epidêmico ocorrido no período de 14 de outubro a 15 de dezembro de 1984 . A

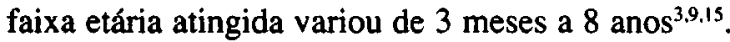
As crianças atingidas apresentaram um quadro inicial agudo de febre alta associada a vômitos e dores abdominais. No período de 12 a 48 horas após o início da febre, desenvolverám púrpura seguida de colapso vascular e necrose de tecidos periféricos ${ }^{1,8,14}$. Conjuntivite purulenta, 3 a 15 dias antes dos sintomas descritos, foi um achado freqüente na maioria dos casos fatais 4 .

De janeiro a maio de 1984, um surto de FPB atingiu a cidade de Londrina-PR ${ }^{7}$. Apesar deste município ter sido atingido primeiro, o diagnóstico definitivo só ocorreu pela revisão dos casos, pois si-

* Baseado na dissertação de Mestrado "Estudo Epidemiológico da Febre Purpúrica Brasileira: Estudo caso-controle: Epidemia em Serrana-SP, 1986", apresentada ao Departamento de Medicina Social da Faculdade de Medicina de Ribeirão Preto, em 1988.

** Departamento de Medicina Social da Faculdade de Medicina de Ribeirão Preto da Universidade de São Paulo - Ribeirão Preto, SP - Brasil.

Separatas/Reprints: L.R.S. Kerr-Pontes - Av. Bandeirantes, 3900 - 14049 - Ribeirão Preto, SP - Brasil.

Publicação financiada pela FAPESP. Processo Medicina 90/4602-1. multaneamente houve um surto de doença meningocócica dificultando a sua caracterização.

Em 28 de março de 1986 teve início no Município de Serrana-SP um surto de FPB". Novamente o quadro clínico de "meningococcemia" apareceu associado à conjuntivite purulenta e, simultaneamente, uma epidemia de conjuntivite, com as mesmas características e sem quadro de FPB associado, atingindo crianças menores de 10 anos de idade.

Uma situação nova ocorreu no Município de Serrana: a incidência de FPB e conjuntivite purulenta foi alta num parque infantil municipal que concentrava cerca de 700 crianças menores de 7 anos. A ocorrência de FPB em grupos aglomerados de até 10 anos de idade era fato inédito até aquele momento.

Até o início do surto em Serrana, o único dado freqüente encontrado foi $o$ isolamento de Haemophylus aegyptius da conjuntiva de crianças com conjuntivite que não tiveram $\mathrm{FPB}^{13}$. Este agente também foi isolado da conjuntiva de crianças nos municípios de Lins (simultâneo ao surto de Promissão, 1984) e de Guariba (simultâneo ao surto de Serrana, 1986), porém nestes não ocorreu (pelo menos não se teve conhecimento) nenhum caso de FPB, neste período. Nenhum agente microbiano havia sido isolado de hemocultura ou líquor de crianças com FPB em Promissão ou Londrina. O Haemophylus aegyptius foi isolado de um raspado de petéquia de pele, colhido sem assepsia, de um caso de FPB em Promissão.

Até esse momento, o $H$. aegyptius era conhecido como o agente causal de surtos de conjuntivite purulenta benigna ${ }^{6.12}$. 
Diante destes fatos, o presente trabalho foi realizado com objetivo de estudar a associação entre FPB e as seguintes variáveis: ocorrência de conjuntivite, aglomeração das pessoas e sintomas respiratórios.

\section{Material e Método}

Foi efetuado um estudo modelo caso-controle.

Definição de Caso. Tomou-se uma série de sintomas e/ou sinais e resultados laboratoriais e atribuiu-se um sistema de pontuação a cada um, além de se utilizar aspectos epidemiológicos ${ }^{10}$. Esta pontuação atribuída se baseou na experiência de vários profissionais que estiveram presentes no atendimento clínico-epidemiológico aos pacientes.

Para cada um dos sinais e/ou sintomas foram atribuídos os respectivos pontos: febre $=5$; diarréia e/ ou vômitos $=1$; fenômenos hemorrágicos $=3$; plaquetopenia e/ou leucopenia $=3$; hemocultura e/ ou líquor e/ou cultura de orofaringe positiva para Haemophilus aegyptius $=7$ e Síndrome de Waterhouse Friedrichsen $=7$. do:

A partir deste sistema de pontuação foi defini-

Caso confirmado: "score" maior ou igual a 12 pontos; caso suspeito: 8 a 12 pontos.

Fonte dos casos: foram todos os casos ocorridos no período de 28/03 a 15/05 durante a epidemia de FPB ocorrida em Serrana-SP, 1986. Esses casos receberam assistência médica na Santa Casa de Serrana; no Hospital das Clínicas da Faculdade de Medicina - USP, no Hospital Santa Lydia e no Hospital São Lucas de Ribeirão Preto.

Controle - Chamou-se de controle, as crianças com "score" menor do que 5 . O critério de seleção foi tomar a primeira criança que satisfizesse as condições de pareamento. As variáveis pareadas foram: sexo, idade e condições sócio-econômicas. Localizou-se o controle tomando-se irmãos e/ou residentes no mesmo local do caso; na impossibilidade de satisfazer este critério foi tomada a primeira criança que se encontrasse na quadra de residência do caso, percorrendo-se esta no sentido horário.

Informaçôes levantadas - O protocolo de investigação que utilizamos foi elaborado no sentido de se obter informações dos casos e controles sobre os seguintes aspectos: identificação do paciente, interrogatório clínico, informações epidemiológicas e dados laboratoriais.

O método estatístico utilizado nas análises dos resultados foi o qui-quadrado com aproximação de Fisher.
Para se estimar o risco de ocorrência da FPB entre crianças matriculadas e crianças não matriculadas na creche municipal, calculou-se o risco relativo (RR). Para tanto utilizou-se a incidência de FPB no grupo exposto ("crianças matriculadas") chamada aqui de "Ie"; e a incidência de FPB no grupo não exposto ("crianças não matriculadas") chamada de "Io". O risco relativo foi então calculado como RR = Ie/lo.

Para os riscos relativos com valores maiores que um, calcularam-se também os intervalos de confiança.

\section{Resultados}

Na Figura 1 são apresentados os casos de FPB (suspeitos e confirmados) ocorridos no período de estudo.

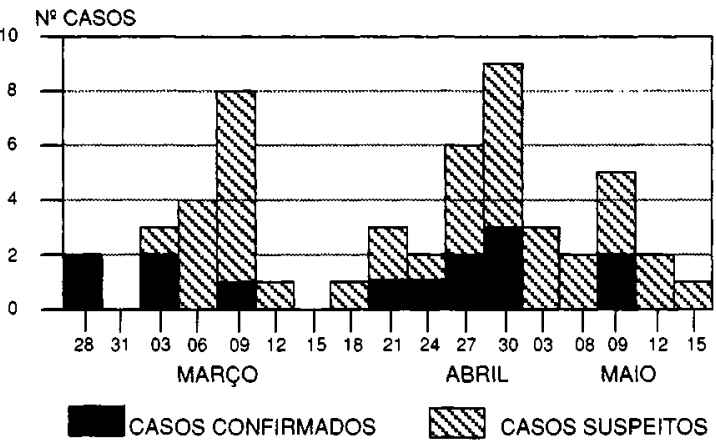

Figura 1. Distribuição dos casos de FPB, segundo a data de ocorrência, Município de Serrana .. SP, 28.03.86 a 15.05.86.

A distribuição dos casos de FPB e seus controles, segundo sexo e idade, são apresentados na Tabela 1. Observa-se o total de 14 casos confirmados, 10 masculinos e 4 femininos, 38 casos suspeitos, 19 masculinos e 19 femininos e um total de 78 controles, 54 masculinos e 24 femininos.

Na Tabela 2 é apresentada a distribuição dos casos confirmados e suspeitos de FPB e dos controles segundo a ocorrência de conjuntivite atual e/ou pregressa encontrando-se os seguintes valores de quiquadrado para os casos confirmados, os suspeitos e para o total de casos, respectivamente: $\mathrm{x}^{2}=11,77$; $\mathrm{x}^{2}=40,91$ e $\mathrm{x}^{2}=44,87$.

Na Tabela 3 é apresentada a distribuição dos casos confirmados e suspeitos de FPB e os controles segundo a ocorrência de sintomas respiratórios. Encontraram-se os seguintes valores do qui-quadrado: para os casos confirmados $\mathrm{x}^{2}=3,28$; para os casos suspeitos $\mathrm{x}^{2}=0,61$ e para o total dos casos $\mathrm{x}^{2}$ $=0,0013$.

A distribuição das crianças e dos casos confirmados e suspeitos de FPB residentes no Município de 
Tabela 1. Distribuição dos casos de Febre Purpúrica Brasileira (FPB) e controles, segundo sexo e faixa etária, Município de Serrana - SP, 28.03.86 a 15.05.86.

\begin{tabular}{|c|c|c|c|c|c|c|c|c|c|c|}
\hline \multirow{2}{*}{$\begin{array}{c}\text { Faixa Etária } \\
\text { (em anos) }\end{array}$} & \multicolumn{3}{|c|}{ Caso Confirmado } & \multicolumn{3}{|c|}{ Caso Suspeito } & \multirow{2}{*}{ Total } & \multicolumn{3}{|c|}{ Controles } \\
\hline & Masc. & Fem. & $\begin{array}{l}\text { Sub- } \\
\text { Total }\end{array}$ & Masc. & Fem. & $\begin{array}{l}\text { Sub- } \\
\text { Total }\end{array}$ & & Masc. & Fem. & Total \\
\hline $0--1$ & 1 & 1 & 2 & 2 & 3 & 5 & 7 & 7 & 3 & 10 \\
\hline $1--2$ & 3 & 1 & 4 & 2 & 3 & 5 & 9 & 7 & 2 & 9 \\
\hline $2--3$ & 0 & 0 & 0 & 3 & 3 & 6 & 6 & 5 & 6 & 11 \\
\hline $3--4$ & 2 & 1 & 3 & 3 & 2 & 5 & 8 & 4 & 2 & 6 \\
\hline $4--5$ & 3 & 0 & 3 & 3 & 3 & 6 & 9 & 5 & 1 & 6 \\
\hline $5--6$ & 0 & 0 & 0 & 3 & 2 & 5 & 5 & 12 & 1 & 13 \\
\hline $6--7$ & 1 & 0 & 1 & 2 & 1 & 3 & 4 & 3 & 2 & 5 \\
\hline $7--+$ & 0 & 1 & 1 & 1 & 2 & 3 & 4 & 11 & 7 & 18 \\
\hline Total & 10 & 4 & 14 & 19 & 19 & 38 & 52 & 54 & 24 & 78 \\
\hline
\end{tabular}

Tabela 2. Distribuição dos casos de Febre Purpúrica Brasileira (FPB) e dos controles, segundo a ocorrência de conjuntivite, atual e/ou pregressa, Município de Serrana SP, 28.03.86 a 15.05 .86 .

\begin{tabular}{lrrrr}
\hline Conjuntivite & \multicolumn{3}{c}{ Casos } & \multirow{2}{*}{ Controles } \\
\cline { 2 - 4 } & Conf. & Susp. & Total & \\
\hline Sim & 11 & 35 & 46 & 21 \\
Não & 3 & 3 & 6 & 57 \\
Total & 14 & 38 & 52 & 78 \\
\hline
\end{tabular}

para casos confirmados : $\mathrm{X}^{2}=11,77$

para casos suspeitos : $X^{2}=40,91$

para total de casos $: X^{2}=44,87$

Tabela 3. Distribuição dos casos de Febre Purpúrica Brasileira (FPB) e dos controles, segundo a ocorrência de sintomas respiratórios, Município de Serrana - SP, 28.03.86 a 15.05 .86 .

\begin{tabular}{ccccc}
\hline \multirow{2}{*}{$\begin{array}{c}\text { Sintomas } \\
\text { Respiratórios }\end{array}$} & \multicolumn{3}{c}{ Casos } & Controles \\
\cline { 2 - 4 } & Conf. & Susp. & Total & \\
\hline Sim & 4 & 26 & 30 & 46 \\
Não & 10 & 12 & 22 & 32 \\
\hline Total & 14 & 38 & 52 & 78 \\
\hline
\end{tabular}

para casos confirmados: $\mathrm{X}^{2}=3,28$

para casos suspeitos : $X^{2}=0,61$

para total de casos : $\mathrm{X}^{2}=0,0013$

Serrana, segundo a variável "estar ou não matriculado no Parque Municipal", é apresentada na Tabela 4. Nesta situação utilizou-se para a análise o risco relativo cujo valor encontrado para os casos confirmados foi de $R R=2,84$, com o intervalo de confiança (IC) de $0,87-8,22$ e para os suspeitos foi de $R R=$ 1,88 e um IC: $0,92-4,06^{2}$.
Tabela 4. Distribuição das crianças e dos casos confirmados e suspeitos de Febre Purpúrica Brasileira (FPB) residentes no município, segundo a variável "estar matriculado ou não" no Parque Municipal, Município de Serrana - SP, 28.03.86 a 15.05.86.

\begin{tabular}{lrrrrrr}
\hline \multirow{2}{*}{$\begin{array}{l}\text { Situação das } \\
\text { crianças }\end{array}$} & \multicolumn{2}{c}{ Casos Confirmados } & & \multicolumn{2}{c}{ Casos Suspeitos } \\
\cline { 2 - 3 } & Sim & Não & & Sim & Não \\
\hline Natriculadas & 5 & 695 & & 10 & 690 \\
\hline Total Matriculadas & 9 & 3.654 & & 24 & 3.635 \\
\hline
\end{tabular}

* 4 casos ignorados

para casos confirmados : Risco Relativo $=2,84$

IC $=0,87--8,22$

para casos suspeitos $\quad$ Risco Relativo $=1,88$

IC $=0,92--4,06$

O total de casos de FPB (confirmados e suspeitos), e das crianças que não tiveram FPB residentes no município, distribuido em 3 períodos de tempo semelhante de acordo com a época de ocorrência dos casos é apresentada na Tabela 5. Encontraramse os seguintes riscos relativos e seus respectivos intervalos de confiança: para o primeiro período $R R$ = 4,19 com IC: 1,41 - 9,30; para o segundo período $\mathrm{RR}=2,42$ com IC: 0,79 - 5,68; e para o terceiro período $R R=0,52$ (neste caso não se calculou $o$ intervalo de confiança por se tratar de um risco menor que 1 ).

A variável tempo (em dias) decorrido entre o início da conjuntivite e a febre (sintoma maior e inicial do quadro clínico da FPB propriamente dita) são apresentados na Figura 2. Calculou-se a mediana (em dias) para os casos confirmados (Mc), casos suspeitos (Ms) e total (Mt), encontrando-se os seguintes resultados: $\mathrm{Mc}=13$ dias; $\mathrm{Ms}=7,3$ dias $\mathrm{e}$ $\mathrm{Mt}=7$ dias. 
Tabela 5. Distribuição das crianças e do total de casos (suspeitos e confirmados) de Febre Purpúrica Brasileira (FPB) residentes no município, segundo a variável "estar matriculado ou não" no Parque Municipal, de acordo com o período de ocorrência dos casos, Município de Serrana - SP, 28.03.86 a 15.05.86.

\begin{tabular}{lrrrrrrr}
\hline Situação das crianças & \multicolumn{7}{c}{ Período de Ocorrência da FPB } \\
\cline { 2 - 7 } & 28.03 & -- & 15.04 & 15.04 & -03.05 & 03.05 & -18.05 \\
& Caso & Não Caso & Caso & Não Caso & Caso & Não Caso \\
\hline Matriculadas* & 8 & 692 & 6 & 694 & 1 & 699 \\
Não Matriculadas* & 10 & 3.653 & $13^{* *}$ & 3.650 & $10^{* *}$ & 3.653 \\
\hline Total & 18 & 4.345 & 19 & 4.344 & 11 & 4.352 \\
\hline
\end{tabular}

1\% período : Risco Relativo $=4,19$

$$
\text { IC }=1,41-9,30
$$

20 período : Risco Relativo $=2,42$

IC $=0,79-5,68$

$3^{2}$ período: Risco Relativo $=0,52$

* 700 crianças matriculadas (Fonte: Parque Municipal de Serrana)

3.663 crianças menores de 9 anos subtraindo-se 700 crianças do parque da população de menores de 9 anos (SEADE: 4.363), Serrana - SP, 1986.

** 2 casos ignorados nos períodos.

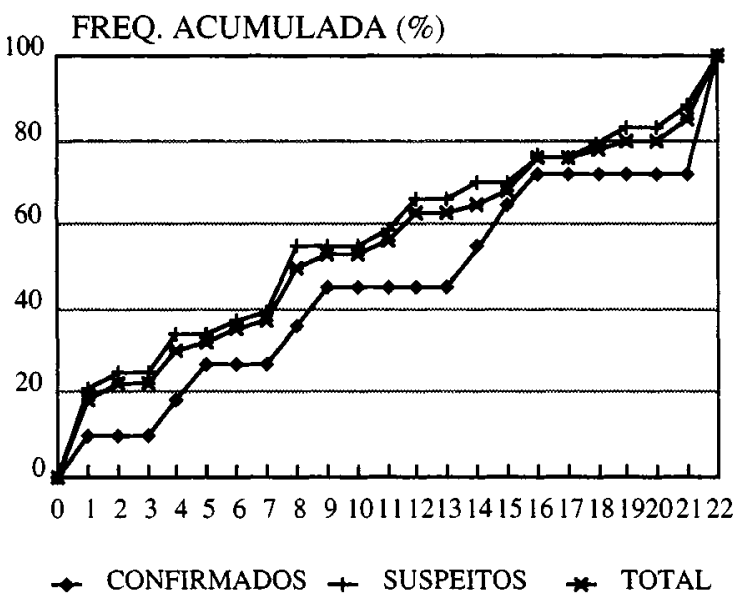

Figura 2. Distribuição dos casos confirmados e suspeitos de Febre Pupúrica Brasileira em frequiências acumuladas, segundo o tempo decorrido entre o início da conjuntivite e a doença, Município de Serrana - SP, 1986.

\section{Discussão}

Na Figura 1 observa-se que a distribuição dos casos de FPB não obedece ao padrão de epidemia por fonte comum parecendo estar mais próximo do padrão pessoa-pessoa. Poderia, também, estar expressando uma transmissão da conjuntivite pessoapessoa e/ou pelo vetor mecânico ou de objetos, que, posteriormente, desencadearia a FPB em algumas crianças, de acordo com a hipótese do trabalho da conjuntivite estar associada à ocorrência de FPB.

Na Tabela 1 observa-se que o número de casos e controles estão restritos às crianças menores de 10 anos. $\mathrm{O}$ número relativamente pequeno de casos no início da epidemia sugeria que o número de controles deveria ser de 3 a 4 para cada caso. No entanto, o número de casos no final do período de estudo permitiu restringir-se esta proporção finalizando-se o trabalho com uma média de 1,5 controle para cada caso.

$\mathrm{Na}$ Tabela 2 observa-se que existe forte associação entre conjuntivite atual e/ou pregressa e a FPB considerando-se os valores dos qui-quadrados encontrados.

Por outro lado, não se observa associação estatisticamente significante entre FPB e sintomas respiratórios como coriza e/ou tosse (Tabela 3). A alta incidência de sintomas respiratórios entre os controles e o número de crianças que foram desprezadas como controle, por terem apresentado febre e sintomas respiratórios 30 dias antes da entrevista, poderiam ser indicativos de que uma virose teria atingido o município simultaneamente à epidemia.

$\mathrm{Na}$ Tabela 4, observa-se que parece haver associação entre aglomeração no parque e casos confirmados e suspeitos de FPB tendo em vista os riscos relativos encontrados.

Durante a epidemia houve uma evasão das crianças matriculadas no parque após a primeira quinzena. Considerando-se este fato relevante em relação ao fator aglomeração, optou-se por estudar a epidemia em 3 períodos distintos apresentados na Tabela 5.

Tomando-se o primeiro período como o mais representativo por caracterizar o início da epidemia e porque as crianças matriculadas ainda estavam freqièentando o parque, observou-se, pelo risco relativo encontrado, que há associação entre aglomeração e FPB. 
Dada a impossibilidade de se obter o número de crianças que frequentaram efetivamente o parque no segundo e terceiro períodos, utilizou-se, para o cálculo da incidência, o número de crianças matriculadas. Este fato poderia ter contribuído para alterar os riscos relativos desses períodos.

A observação da existência de associação entre FPB e conjuntivite purulenta atual e/ou pregressa, influenciou ativamente a prática da vigilância epidemiológica da FPB no Estado de São Paulo. Em 1986 quando se deu o início do surto em Serrana, a vigilância epidemiológica da FPB dava-se pela investigação de casos de doença meningocócica sob a forma de meningococcemia, conforme já foi assinalado. Sabe-se, hoje, que a FPB quando diagnosticada precocemente tende a uma evolução benigna e que o quadro de manifestações hemorrágicas que ocorre na fase final pode ser evitado $^{5,16}$

A importância da conjuntivite como patologia que frequientemente precede a instalação do quadro clínico da FPB e as observações de sua associação com a FPB merecem algumas considerações que se farão a seguir.

Pesquisadores do Instituto Adolfo Lutz (IAL)* produziram um anti-soro a partir de cepas isoladas de casos confirmados de FPB que se aglutina em presença de cepas de Haemophilus aegyptius causadores de FPB possibilitando um diagnóstico presuntivo deste agente, em nível dos laboratórios regionais, antes de sua completa, e mais lenta, identificação laboratorial em nível central. Esta prática poderá ser muito útil em nível dos serviços locais e regionais para diagnóstico precoce de identificação do agente dos casos de conjuntivite de ocorrência, num determinado município.

Esta técnica desenvolvida pelo IAL de reconhecimento presuntivo das cepas de Haemophilus causadores de FPB, a associação da conjuntivite com a FPB e as medidas de tempo em dias encontradas entre $o$ início da conjuntivite e o início da FPB poderá ser de grande utilidade na prática da vigilância epidemiológica da FPB.

De acordo com os resultados das medianas acima citadas, conclui-se que se tem um período de uma a duas semanas para o reconhecimento da cepa patogênica incidente num determinado município para que se consiga prevenir os possíveis casos de FPB que possam vir a ocorrer.

A prevenção da FPB se dará com a orientação adequada dos profissionais da saúde responsáveis pela assistência médica no município, seja através de serviços públicos, credenciados ou particulares.

Cabe ainda ressaltar que este trabalho de diag-

* Informação pessoal. nóstico da situação epidemiológica do município, a prevenção, a orientação e a normatização das condutas a serem tomadas, deve ser realizado pela Vigilância Epidemiológica do Escritório Regional de Saúde, responsável pela área atingida.

\section{Consideraçóes gerais}

A investigação da epidemia de FPB em Serrana envolveu instituições como Secretaria Estadual da Saúde, Faculdade de Medicina de Ribeirão Preto USP (FMRP - USP), Hospital das Clínicas da FMRP-USP e Santa Casa de Serrana. O trabalho em conjunto com diferentes instituições contribuiu de maneira decisiva no diagnóstico clínico e laboratorial precoce, tratamento eficaz, estabelecimento de uma rotina de referência e, finalmente, a realização da presente investigação.

Em geral, a Secretaria da Saúde precisa responder rapidamente às situações de agravo à saúde e, às vezes, não consegue sistematizar uma investigação de qualidade que permita uma pesquisa científica de hipóteses. Em outro extremo, com freqüência, a Universidade se omite de conhecer a realidade em que vive a população na qual está inserida, o que a nosso ver é lastimável (para a Saúde Pública e para a Universidade). A integração que se verificou entre as instituições que assistiram ao surto de FPB, no município de Serrana, destacando-se a Universidade e a Secretaria da Saúde, foi extremamente relevante para o avanço das investigações desta nova patologia. A academia voltada aos interesses e necessidades da comunidade contribuiu, neste caso, para diminuição significativa da morbidade e mortalidade pela FPB.

KERR-PONTES, L.R.S. \& RUFFINO-NETTO, A. [Brazilian purpuric fever - An epidemiological study of an outbreak in the locality of the S. Paulo State, Brazil, in 1986]. Rev. Saúde públ., S. Paulo, 25: 375-80, 1991. A case control model was used in the study of an outbreak of Brazilian purpuric fever BPF which occurred in Serrana, S. Paulo State, Brazil, in 1986. Three hypotheses were raised: 1 - purulent conjunctivitis is associated with BPF; 2 - a cluster effect accurs in BPF; 3 - respiratory symptoms may be a variation of the clinical picture of the disease. Numerical values were attributed to different findings, as follows: fever $=5$; diarrhea and $/$ or vomiting $=1$; haemorrhagic findings $=3$; thrombocytopenia and/or leukopenia $=3$; Haemophilus aegyptius positive hemoculture and/or Haemophilus aegyptius positive cerebrospinal fluid culture and/or $H$. $a$. oropharynx culture $=7$; Waterhouse Friedrichsen syndrome $=7$. Those cases for which the sum total of points reached or exceeded 13 were considered as confirmed and those obtaining between 8 and 12 were considered as suspect. Children with a score below 5 were taken as control cases. Cases and controls were matched according to sex, age and socioeconomic level. The to- 
tal groups studied included 14 confirmed cases, 38 suspect cases and 78 controls. It was concluded that purpuric fever is strongly associated with previous and/ or present purulent conjunctivities; a cluster effect seems to occur; respiratory symptoms such as coughing and/or coryza were not associated with BPF.

Keywords: Waterhouse-Friederichsen syndrome, epidemiology. Disease outbreaks.

\section{Referências Bibliográficas}

1. ASPECTOS clínicos e epidemiológicos de 14 casos da FBP com Haemophylus aegyptius no sangue. Bol. Centro Vigil. epidem., S. Paulo, 1 (19) out. 1986.

2. BAILEY, B.J.R. Confidence limits to the risk ratio. Biometrics, 43 (1): 201-6, 1987.

3. BOLETIM EPIDEMIOLÓGICO. Brasília, Ministério da Saúde, 17 (1/2) 1985.

4. BRAZILIAN PURPURIC FEVER STUDY GROUP. Brazilian Purpuric Fever: epidemic purpura fulminash associated with antecedent purulent conjuctivitis, Lancet, 2: 757-61, 1987.

5. BRAZILIAN PURPURIC FEVER STUDY GROUP Haemophilus aegyptius bacteraemia in Brazilian Purpuric Fever. Lancet, 2: 761-3, 1987.

6. BUEHLER, J.W.; HOLLOWAY, I.T.; GOODMAN, R.A. SIKES, R.K. Gnat Sore Eyes: seasonal acute conjunctivitis in a Southern State. South med. J., 76: 587 9, 1983.

7. CAMARGO, H.; BALDY, J.L.S.; DAL MOLIN, E.M.C. Febre purpúrica brasileira em Londrina, Paraná. Bol. epidem. Paraná, 5 (51) 1986.

8. CENTERS FOR DISEASE CONTROL. Brazilian Purpuric fever Haemophylus aegyptius bacteremia complicating purulent conjunctivitis. Morb. Mort. wkly Rep., 35: 553-4, 1986.

9. CENTERS FOR DISEASE CONTROL. Preliminary report: epidemic fatal purpuric fever among children - Brazil. Morb. Mort. wkly Rep., 34: 217-9, 1985.

10. GERMANO NETO, J. Aspectos epidemiológicos dos casos e suspeitas em Serrana. In: Silva, G.A. Relatório do Seminário sobre Febre Purpúrica do Brasil. São Paulo, Secretaria de Estado da Saúde. Centro de Vigilância Epidemiológica, 1986. p. 35-45.

11. GERMANO NETO, J. \& KERR-PONTES, L.R.S. Febre Purpúrica do Brasil (FPB), em Serrana, São Paulo, Bol. epidem., Brasília, 18 (17/20): 93-103, 1986.

12. IRINO, K. Análise dos aspectos da microbiologia. In: Silva, G.A. Relatório do Seminário sobre Febre Purpúrica do Brasil. São Paulo, Secretaria de Estado da Saúde. Centro de Vigilância Epidemiológica, 1986. p.55-61.

13. IRINO, K.; LEE, I.M.L.; KARU, M.; BRANDILEONE, M.C.C.; MELLES, C.E.A.; LEVY, C.M.; BERKLEY, S.E.; FLEMING, D.W.; SILVA, G.A.; HARRISON, L. Febre Purpúrica Brasileira: resultados preliminares da Investigação Etiológica. Rev. Inst. Med. trop., S. Paulo, 29: 174-7, 1987.

14. Mc INTYRE, P.; WHEATON, G.; ERLICH, J. Brazilian Purpuric Fever in Central Australia. Lancet, 2: $112,1987$.

15. MILSTEIN, T. Doença indeterminada na cidade de Promissão: nota prévia. Rev. paul. Med., 103 (2): 95-8, 1985.

16. ROCHA, G.M. Aspectos clínicos: experiência em Ribeirão Preto. In: Silva, G.A. Relatório do Seminário sobre Fe. bre Purpurica do Brasil. São Paulo, Secretaria de Estado da Saúde. Centro de Vigilância Epidemiológica, 1986. p. 27-34.

Recebido para publicação em 06/12/1990

Reapresentado em 21106/1991.

Aprovado para publicaçấo em 0110711991 . 